\title{
BRONZEZEITLICHE GRÄBER MIT ROHMETALL: EINE FUNDGRUPPE ZWISCHEN PRAKTISCHER FUNKTION UND SYMBOLISCHER BEDEUTUNG
}

\author{
A L B R E C H T J O C K E N H ÖV E L
}

\begin{abstract}
Bronze Age Burials with Raw Metal: a Group of Finds Between Practical Function and Symbolical Meaning. In Bronze Age burials objects from the sector of metal craft are very rare. They include raw metal, such as copper bronze ingots of different shapes and bars, only found in about 35 graves exclusively in southern Central Europe, and there especially in the Alps. This tradition starts in the Middle Bronze Age (BB-BC) and reaches its peak in the earlier Urnfield period (BD-HA). The combination of raw metal and further objects connects these burials with the male sphere with one exception (Marzoll - No. 5). Most of them are richly equipped with weapons (swords, spearheads, daggers etc.) and horse-drawn wagons. The relatively frequent combination with sickles and weights points to agricultural activities and a standardized value system. The concentration of raw metal burials near Eastern Alpine copper ore deposits (Tirol, Salzburg) may indicate the control and distribution of the raw metal by warriors and 'traders'.
\end{abstract}

Keywords: Central Europe, Bronze Age, burials, raw metal, alpine copper ore deposits, male sphere, wariors, traders.

Dem Jubilar verdankt die europäische Forschung grundlegende und anregende Beiträge zur Geschichte des bronzezeitlichen Handwerks. Sein besonderes Interesse galt dem Handwerker als Individuum, wie er in den sog. Handwerkergräbern sichtbar wird. Auf seinen Studien und Ergebnissen konnte ich unlängst aufbauen (Jockenhövel 2018). Mein Beitrag soll eine kleine Ergänzung zu diesem Themenbereich sein, wobei ich mich einer „unscheinbaren", aber für die chaîne opératoire im Metallhandwerk wesentlichen Fundgattung widme, dem Rohmetall und wie es als Grabbeigabe verstanden werden kann (erste Arbeiten: Jockenhövel 1973; Hansen 1991, 140 ff.; 1994, 230 ff.).

Rohmetall ist besonders in den jungbronzezeitlichen Hortfunden, wie in den früh- und älterjungbronzezeitlichen Brucherzfunden des Karpaten- und Donaugebietes mit ihrem hohen Anteil an Gusskuchen, eine "gewichtige" Fundgruppe (Nessel 2017). In deutlichem Kontrast dazu sind Gusskuchen und Barren gemessen an ihrer wirtschaftlichen Bedeutung in bronzezeitlichen Grabfunden völlig unterrepräsentiert (vgl. Statistik: Jockenhövel 2018, 219, Abb. 1; 2019b, 11, Abb. 1).

Im Folgenden werden die (Stand 2019) mir erreichbaren Gräber zusammengestellt, die in irgendeiner Form Beigaben enthalten, die insgesamt unter dem Begriff Rohmetall zusammengefasst und von Fertigobjekten unterschieden werden können. Es sind plankonvexe oder fladenförmige Gusskuchen, amorphe Rohmetallbrocken, unterschiedlich geformte Barren und Metallschrott (Tabelle 1). Die Tabelle enthält in alphabetischer Reihenfolge mit laufender Nummer (gekennzeichnet mit * sind für Autor unsichere, grabaffine und/oder unbekannte Grabkontexte) Fundort, Bestattungsart, Fundkombinationen und weiterführende Literatur (Tabelle 1).

Die wenigen Rohmetallstücke in den Gräbern sind von sehr variabler Gestalt (Abb. 1). Die Bruchstücke von Ederheim/Hügel 8 (Nr. *2), Barbing (Nr. 1), Marzoll (Nr. 5), Milavče/Hügel C/4 (Nr. 6), Poing/Grab 1 (Nr. 7), Volders/Gräber 256 und 390 (Nr. 9, 10) wurden von ehemals vollständigen plankonvexen Gusskuchen abgetrennt, die ab der mittleren Bronzezeit eine eigene „zeitlose“ Fundgruppe darstellen (vgl. Abb. 1: $1,2,5,6,9)$. Die Fragmente sind nur noch der letzte Rest ehemals größerer und kilogrammschwerer Stücke (Bachmann u. a. 2003, 81 ff., 107, Abb. 18; Modl 2010; 2019; Nessel 2017). Vollständige Gusskuchen, die ein erhebliches Gewicht haben können und somit einen hohen Materialwert besitzen (z. B. Bachmann u. a. 2003, 106, Tabelle 8), sind aus Gräbern nicht überliefert. Die meisten Gussbrocken entziehen sich jedoch einer formalen Ansprache; sie dürften aus unterschiedlichen Portionierungsvorgängen stammen (Modl 2019; Nessel 2017, 179, Abb. 8; Reiter/Linke 2016, $150 \mathrm{ff} ., \mathrm{Abb}$. 22). Nach den bisher wenigen vorliegenden Analysen bestehen einige südbayerische und nordtirolische Gusskuchen und Gussbrocken aus Gräbern aus weitgehend spurenfreiem, d. h. aus sehr reinem Rohkupfer (vermutlich aus Kupferkieserzen, wie Poing/Grab 1 - Nr. 7) oder aus fahlerzgeprägtem Kupfer (d. h. mit prägnanten Spuren von Arsen, Antimon und Nickel, wie Barbing-Nr. 1, Volders-Nr. 9). Kleinere und leichtere fladenförmige Gussbrocken könnten aus Zinnbronze bestehen, somit Produkte eines vielfach von der Forschung angenommenen 
Tabelle 1. Rohmetall in Gräbern. Abkürzungen: 1: A - Axt; Am - Amboss; B - Brandbestattung; Ba - Barren; BG - Bronzegefäß; Bro-Bronze; $\mathrm{Cu}$-Kupfer; D - Dolch; Dep-Depot in Grabhügel; DK-Doppelknopf; Fa - Fahlerzkupfer; FH - Fleischhaken; G-Gewicht; g-Gramm; Hgl. - Grabhügel; K - Körperbestattung; L - Lanze; M - Messer; MS - Meißelschneide; N - Nadel; $\mathrm{Pb}$ - Blei; Pd - Pferd; Pf-Pfeil; Pfr-Pfriem/Ahle; Pic-Pickel; Pin-Pinzette; RM-Rasiermesser; S-Schwert; Sch-Schmuck; Si - Sichel; Sn - Zinn; W - Wagen (Autoren A. Jockenhövel, K. Zehr-Milić).

\begin{tabular}{|c|c|c|c|c|c|c|c|c|c|c|c|c|c|}
\hline 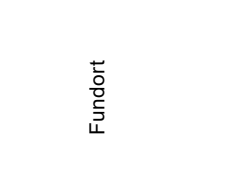 & 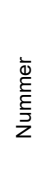 & 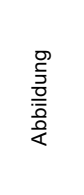 & 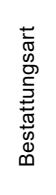 & 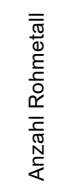 & 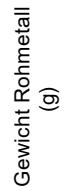 & 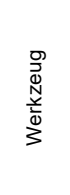 & 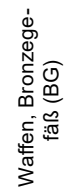 & 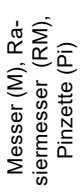 & 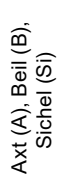 & 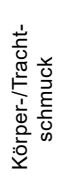 & 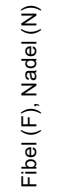 & 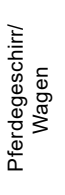 & 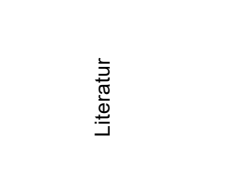 \\
\hline \multicolumn{14}{|l|}{$\begin{array}{l}\text { Plankonvexe/fladenför- } \\
\text { mige Gusskuchen }\end{array}$} \\
\hline Barbing & 1 & $1,1 a-b$ & B & $2(\mathrm{Fa})$ & - & - & - & - & - & - & $\mathrm{N}$ & - & Hennig 1993 \\
\hline Ederheim Hgl. 8 & *2 & 1,2 & $\mathrm{~K}$ & 1 & - & - & - & Pin & $4 \mathrm{Si}$ & - & - & - & Ludwig-Lukanow 1983 \\
\hline Ilvesheim & 3 & 1,3 & $\mathrm{~K}$ & 1 & 35 & - & $D$ & - & A & $x$ & - & - & Görner 2003 \\
\hline Jüchsen & 4 & $\begin{array}{c}1 \\
4 a-b\end{array}$ & $\mathrm{D}$ & 2 & 245 & - & - & - & B & - & - & - & Feustel 1993 \\
\hline Marzoll & 5 & 1,5 & B & 5 & 537 & - & - & M & - & - & $2 \mathrm{~N}$ & - & Hell 1948 \\
\hline Milavče C/4 & 6 & 1,6 & B & 1 & 25,07 & G & $S, L, P$ & M & - & $\mathrm{x}$ & SN & $\begin{array}{l}W \\
P d\end{array}$ & Pare 1999 \\
\hline Poing 1 & 7 & 1,7 & B & 1 & - & $\mathrm{Ba}, \mathrm{G}$ & $\begin{array}{l}\mathrm{S}, \mathrm{Pf} \\
\mathrm{BG}\end{array}$ & RM & $\mathrm{Si}$ & $\mathrm{x}$ & $2 \mathrm{~N}$ & - & Clausing 2005 \\
\hline Straubing 26 & 8 & 1,8 & B & 1 & 36,6 & - & - & $\mathrm{FH}$ & - & $\mathrm{x}$ & $\mathrm{N}$ & - & Hundt 1964 \\
\hline Volders 256 & 9 & $\begin{array}{c}1 \\
9 a-b\end{array}$ & B & $2(\mathrm{Fa})$ & - & - & - & M & - & - & $\mathrm{N}$ & - & Kasseroler 1959 \\
\hline Volders 390 & 10 & $\begin{array}{c}1 \\
10 a-b\end{array}$ & B & 1 & - & - & - & M & - & - & $\mathrm{N}$ & - & Kasseroler 1959 \\
\hline Vomp 3 & 11 & $\begin{array}{c}1, \\
23 a-b\end{array}$ & B & - & $\sim 120$ & - & $2 \mathrm{DK}$ & RM, M & - & - & - & - & $\begin{array}{l}\text { Unpubl. (W.Sölder, } \\
\text { TLM Innbruck) }\end{array}$ \\
\hline $\begin{array}{l}\text { Amorphe Rohmetall- } \\
\text { stücke }\end{array}$ & & & & & & & & & & & & & $\begin{array}{l}\text { Amorphe Rohme- } \\
\text { tallstücke }\end{array}$ \\
\hline Hallstatt 797 & 12 & $\begin{array}{c}1, \\
11 a-b\end{array}$ & $\mathrm{~K}$ & 2 & - & $\mathrm{Pic}$ & - & - & - & - & - & - & Kromer 1959 \\
\hline Ederheim Hgl. 30 & *13 & 1,12 & $?$ & 1 & - & - & - & - & - & $x$ & - & - & Ludwig-Lukanow 1983 \\
\hline Horušany & 14 & $\begin{array}{c}1, \\
13 a-c\end{array}$ & B & 2 & - & $\mathrm{Pfr}, \mathrm{G}$ & - & - & - & $\mathrm{x}$ & - & - & Pare 1999 \\
\hline Königsbronn & 15 & 1,14 & $\begin{array}{l}\text { B/ } \\
\text { Dep }\end{array}$ & 1 & 51 & G & L & - & - & $x$ & $\mathrm{~N}$ & W.Pd & Pankau 2013b \\
\hline $\begin{array}{l}\text { Königswieser Forst } \\
\text { Hgl. } 10\end{array}$ & 16 & 1,15 & $\mathrm{~K}$ & 1 & 24 & - & - & - & - & - & $\mathrm{N}$ & - & Koschik 1981 \\
\hline $\begin{array}{l}\text { Königswieser Forst } \\
\text { Hgl. } 24\end{array}$ & *17 & 1,16 & $\mathrm{~K}$ & 1 & 24,7 & - & - & - & - & - & - & - & Koschik 1981 \\
\hline Kühndorf & 18 & 1,17 & $\mathrm{~K}$ & 1 & 523 & - & $\mathrm{Pf}$ & - & B & $x$ & $\mathrm{~N}$ & - & Ebner 2001 \\
\hline $\begin{array}{l}\text { München-Unterhaching } \\
30\end{array}$ & 19 & 1,19 & B & 1 & 200 & - & S & - & $\mathrm{Si}$ & - & $3 N$ & - & Clausing 2005 \\
\hline Regensburg-Weichs 3 & 20 & 1,20 & B & 2 & - & - & - & - & - & - & $>2 \mathrm{~N}$ & - & Hennig 1993 \\
\hline Rothenstein & *21 & 1,21 & $?$ & 1 & 21,58 & - & - & - & - & - & $3 N$ & - & Wiegel 1992 \\
\hline Volders 239 & 22 & 1,22 & B & 1 & - & - & - & - & - & - & - & - & Kasseroler 1959 \\
\hline Fragliche Gräber & & & & & & & & & & & & & Fragliche Gräber \\
\hline Aberzhausen & 23 & - & $\mathrm{K}$ & 1 & "40“ & - & - & M & $\mathrm{Si}$ & $x$ & N? & - & Berger 1984 \\
\hline Eberfing & 24 & - & $B$ & 1 & - & - & - & M & - & $\times 3 \mathrm{~N}$ & - & - & Koschik 1981 \\
\hline
\end{tabular}


Tabelle 1. Fortsetzung.

\begin{tabular}{|c|c|c|c|c|c|c|c|c|c|c|c|c|c|}
\hline 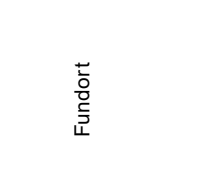 & 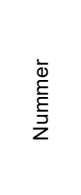 & $\begin{array}{l}\frac{0}{5} \\
\text { 흔 } \\
\frac{\overline{0}}{2}\end{array}$ & 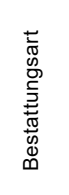 & 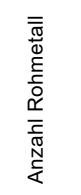 & 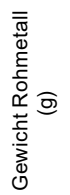 & 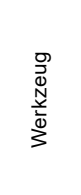 & 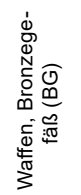 & 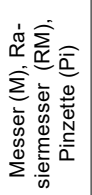 & 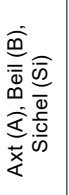 & 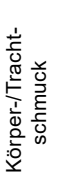 & 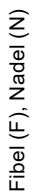 & 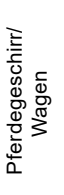 & 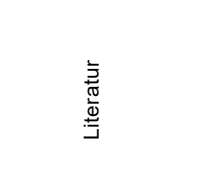 \\
\hline Feldmoching & ${ }^{*} 25$ & - & $?$ & 1 & - & - & - & - & - & - & - & - & Koschik 1981 \\
\hline Möckmühl & 26 & $\begin{array}{c}1, \\
18 a-b\end{array}$ & B & 3 & - & - & s & $2 \mathrm{M}$ & $\mathrm{Si}$ & $\mathrm{x}$ & N & - & Clausing 2005 \\
\hline Volders 305 & 27 & - & B & $4(\mathrm{Fa})$ & - & - & - & - & - & - & - & - & Kasseroler 195 \\
\hline Weischau & ${ }^{*} 28$ & 1,24 & $\mathrm{~K}$ ? & 1 & 345 & - & D & - & $\mathrm{B}, \mathrm{Si}$ & $\mathrm{x}$ & $N$ & - & Berger 1984 \\
\hline Barren & & & & & & & & & & & & & Gräber mit Barren \\
\hline Bomhöved & *29 & - & $\mathrm{K}$ ? & 1 & - & - & - & - & - & - & - & - & $\begin{array}{c}\text { Schwerin von Krosigk } \\
1976\end{array}$ \\
\hline Korntal-Münchingen & 30 & 2,1 & B & $1(S n)$ & - & - & $2,5 \mathrm{~L}$ & - & $\mathrm{Si}$ & $x$ & - & - & Clausing 2005 \\
\hline Kronberg & *31 & 2,1 & Dep? & 1 & - & MS, Pf & $2 \mathrm{~L}, 13$ & - & - & - & - & w & Adler 1990 \\
\hline Lachen-Speyerdorf & 32 & 2,3 & B & $1(\mathrm{~Pb})$ & $>660$ & $\mathrm{Am}$ & - & $\begin{array}{l}2 \mathrm{RM}, \\
\mathrm{M}\end{array}$ & - & - & - & - & Sperber 2000 \\
\hline Poing 1 & $33(=7)$ & 2,4 & B & - & - & - & - & - & - & - & - & - & siehe Nr. 7 \\
\hline Sémoutiers & *34 & - & B & $1(\mathrm{Sn})$ & - & - & - & - & - & - & - & - & Pare 1999 \\
\hline Völs & *35.36. & $2,5-6$ & B & 2 (Bro) & $\sim 480$ & - & - & - & - & - & - & - & Jockenhövel 1973 \\
\hline
\end{tabular}

Recycling-Prozesses sein (z. B. Mozsolics 1981), der aber in seiner Bedeutung im bronzezeitlichen Metallumlauf überschätzt wird (Bachmann u. a. 2003, 108 ff.).

Die Gewichte der Gusskuchen- und Gussbrocken betragen meist nur zwischen 20-50 g, nur wenige wiegen mehr (Tabelle 1: Nr. 4, 5, 11, 19, *28).

Die Stab- und Stangenbarren aus Gräbern (Abb. 2) gehören zu einer recht heterogenen Gruppe von gegenüber den plankonvexen Barren wesentlich leichteren (im Mittel ca. 50-200 g) und kleinformatigen Rohmetallform, die in unterschiedlicher Gestalt ab der Mittelbronzezeit aus zahlreichen Hortfunden Mitteleuropas überliefert sind (Drescher 1976; Mozsolics 1984, 32 f.; Primas/Pernicka 1998). Sie sind -je nach Bedarf - gezielter zerteilbar als die Gusskuchen. Metallanalysen von Barren zeigen eine breite Mischung, die von Rohkupfer über ",klassische“ Zinnbronze zu eigenartigen Mischungen mit zum Teil hohen Zinn-, Blei- und Antimonanteilen reicht. Möglicherweise dienten sie als Vorlegierung oder Beimischungen zur Zinnbronze (Bachmann/Jockenhövel 1974, 142; Salaš 2018, 108). Der sekundär als Amboss verwendete Doppelspitz-Barren aus Lachen-Speyerdorf (Nr. 34; Abb. 2: 3) besteht aus reinem Blei (Bachmann u. a. 2003, 92 f., Abb. 13 mit Verbreitungskarte; Sperber 2004) und ist bisher das einzige Exemplar, das in einem Grab gefunden wurde.

\section{ZEITSTELLUNG UND VERBREITUNG}

Im Folgenden wird schematisch die Zeitstellung der datierbaren Kontexte mit Rohmetall angegeben und auf eine Detaildiskussion verzichtet.

Frühe Mittelbronzezeit (BB): *Feldmoching, Ilvesheim,

Königswieser Forst (Hügel 10, *Hügel 14)

Mittlere und späte Mittelbronzezeit (BC/Periode II):

${ }^{*}$ Ederheim (Hügel 8 und 30), Jüchsen, Kühndorf,

*Weischau

Frühe und ältere Urnenfelderzeit (BD/HA1/Periode III):

*Bornhöved, Poing (Grab 1), Eberfing, Horušany,

Königsbronn, Korntal-Münchingen, *Kronberg,

Milavče (Hügel C/4), *Sémoutiers (?), Straubing (Grab 26)

Mittlere bis Jüngere Urnenfelderzeit (HA2-HB1):

Aberzhausen, Barbing, Lachen-Speyerdorf, Marzoll (Grab 2), Möckmühl, München-Unterhaching (Grab 30), Regensburg-Weichs (Grab 3), Volders (Gräber 239, 256, 305), Vomp (Grab 3)

Urnenfelderzeit, allgemein: *Völs

Späte Urnenfelderzeit (HB3/Periode V): keine Gräber Ältere Eisenzeit (HC/D): Hallstatt (Grab 797)

Grabfunde liegen seit der beginnenden Mittelbronzezeit mit den süddeutschen Funden von Ilvesheim, *Feldmoching, Königswieser Forst 


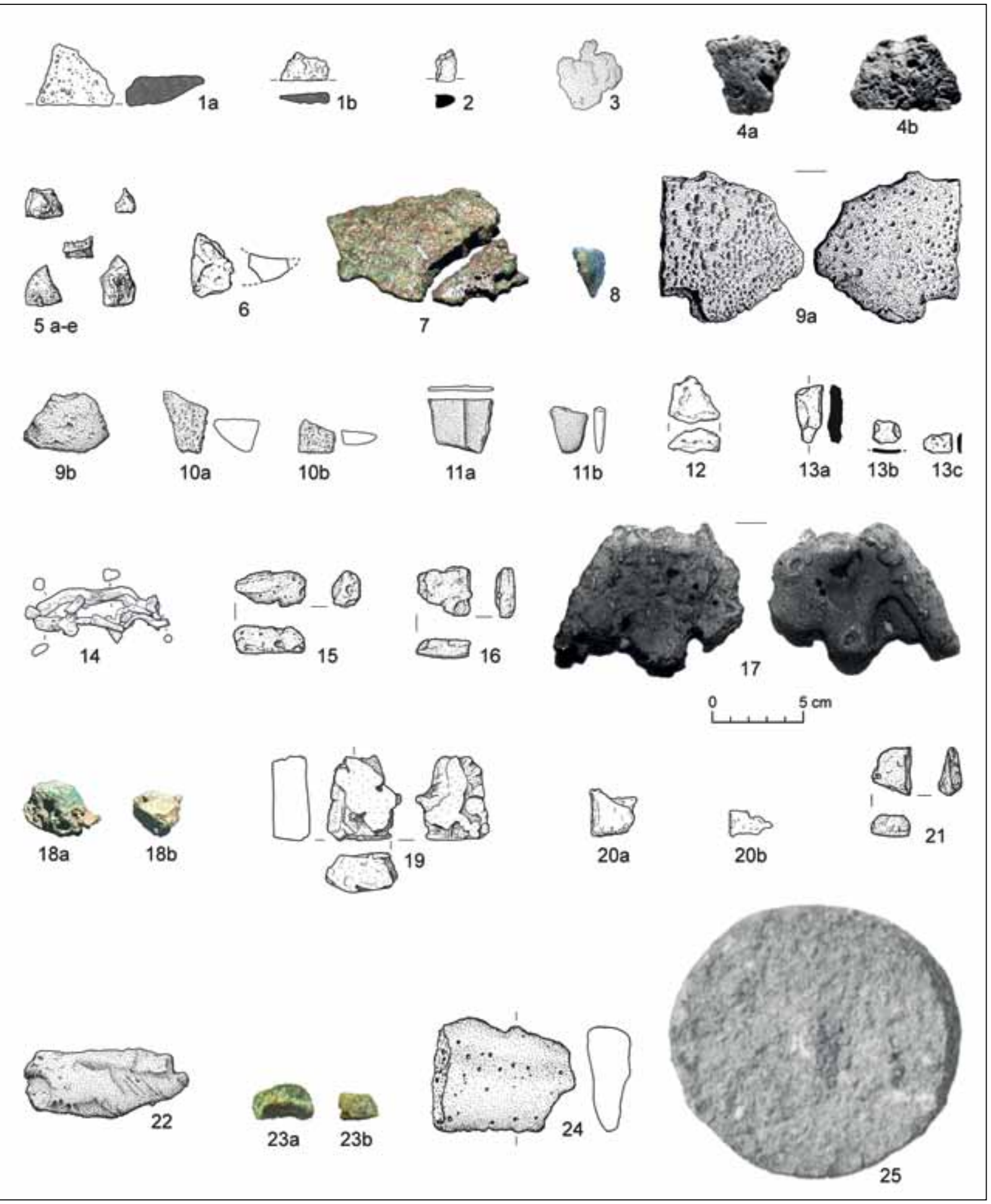

Abb. 1. Gräber mit Rohmetall (Gusskuchen und Gussbrocken). 1a, 1b - Barbing (D); 2 - Ederheim (D), Hügel 8; 3 - Ilvesheim (D); 4a, 4b - Jüchsen (D); 5a-e - Marzoll (A); 6 - Milavče (CZ), Hügel C/4; 7 - Poing (D), Grab 1; 8 - Straubing (D); 9a, 9b - Volders (A), Grab 256; 10a, 10b - Volders (D), Grab 390; 11a, 11b - Hallstatt (A), Grab 797; 12 - Ederheim (D), Hügel 30; 13a-c - Horušany (CZ); 14 - Königsbronn (D); 15 - Königswieser Forst (D), Hügel 10; 16 - Königswieser Forst (D), Hügel 24; 17 - Kühndorf (D); 18 a, 18b - Möckmühl (D); 19 - München-Unterhaching (D), Grab 30; 20a, 20b - Regensburg-Weichs (D), Grab 3; 21 - Rothenstein (D); 22 - Volders (A), Grab 239; 23a, 23b - Vomp (A), Grab 3; 24 - Weischau (D); 25 - Gordion (TR). 1-24-Kupfer/Bronze; 25 - Eisen. Maßstab 1:3 (Autoren A. Jockenhövel, K. Ebner, G./A. Körte, C. Pankau, C. Pare, L. Sperber, H.-P. Uenze, K. Zehr-Milić). 


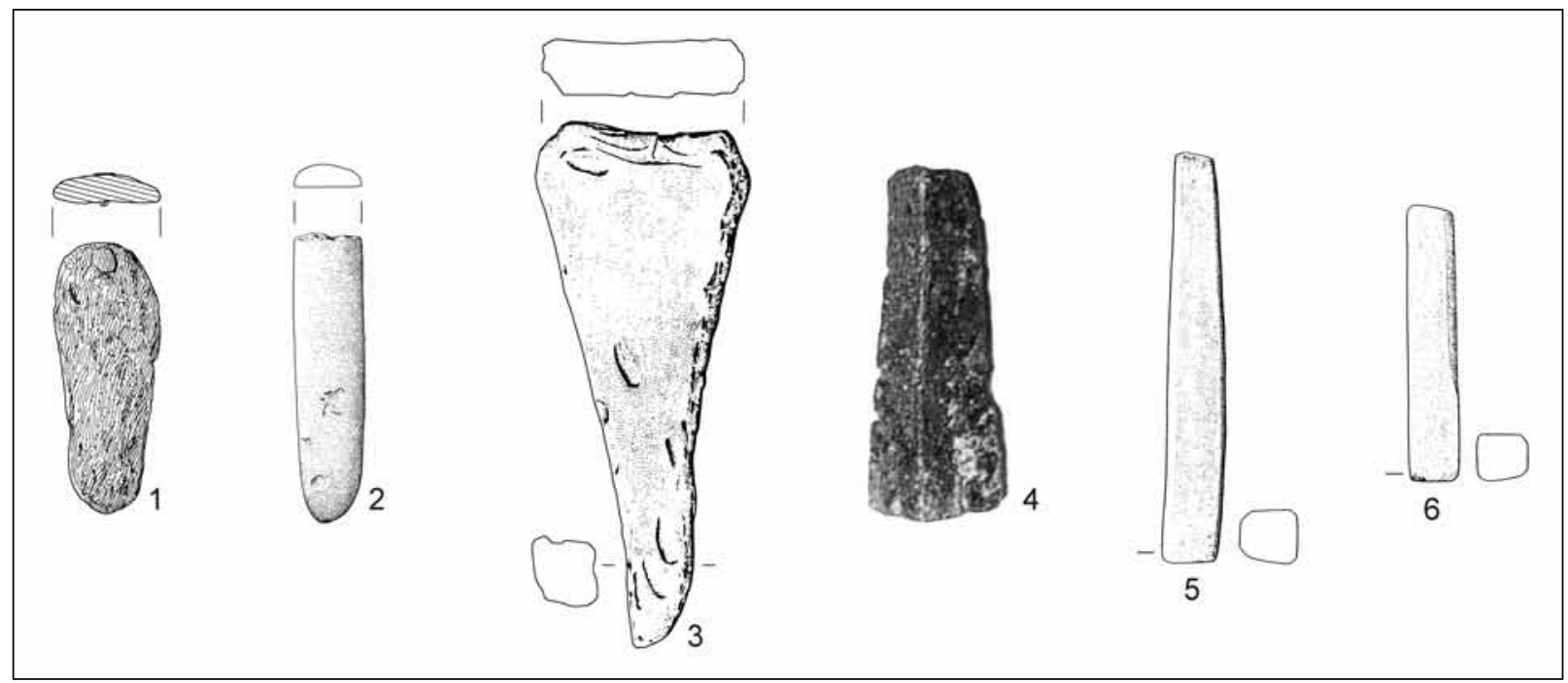

Abb. 2. Gräber mit Barren. 1 - Kronberg (A); 2 - Korntal-Münchingen (D); 3 - Lachen-Speyerdorf (D); 4 - Poing (D), Grab 1; 5, 6 - Völs (A). 1, 2, 4-6-Kupfer/Bronze; 3 - Blei. Maßstab 1:3 (Autoren A. Jockenhövel, L. Sperber, K. Zehr-Milić).

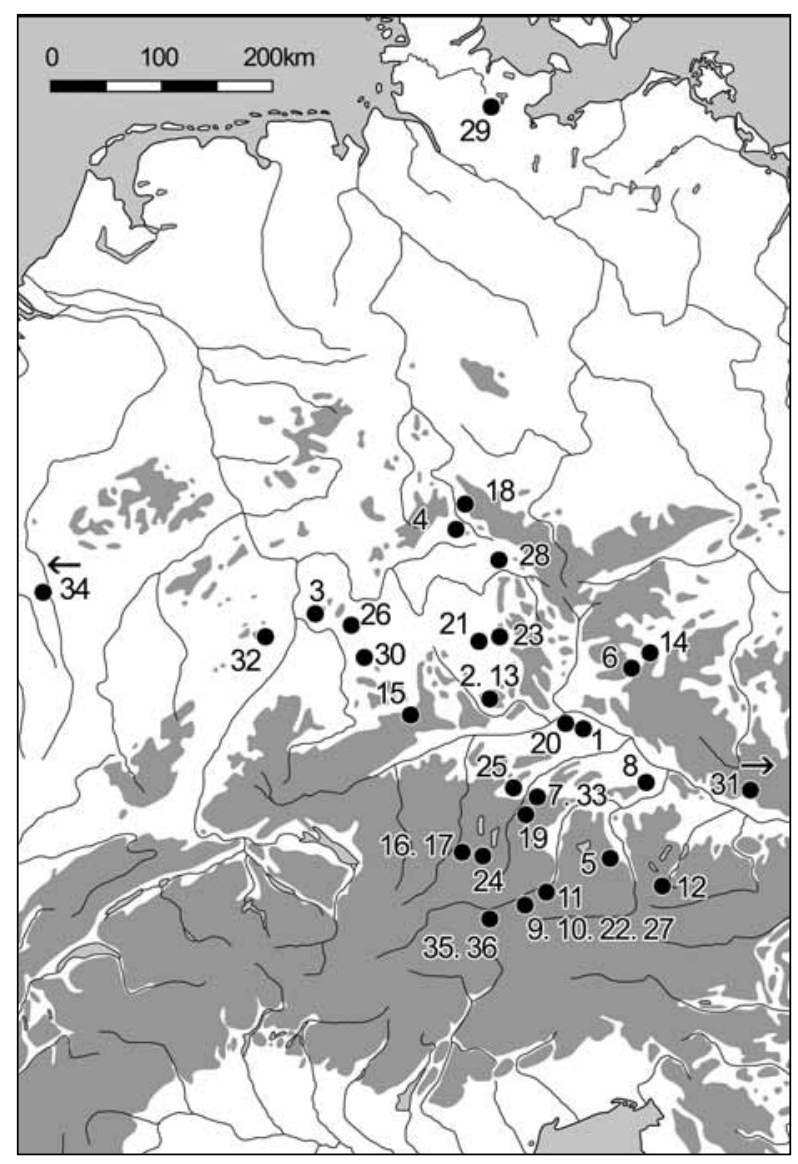

Abb. 3. Verbreitungskarte der bronzezeitlichen Gräber mit Rohmetall (Nummerierung folgt Tabelle 1; Autoren A. Jockenhövel, K. Zehr-Milić).
(Hügel 10), *Ederheim (Hügel 8 und 30), Jüchsen, Kühndorf und *Weischau vor. In die nordische Periode II/III könnte der mutmaßliche Grabfund mit einem Zinnbarren von *Bornhöved (Schleswig-Holstein) datieren. Einen deutlichen zeitlichen und räumlichen Schwerpunkt bilden die zumeist reich ausgestatteten Krieger-/Wagengräber vom Typ Poing/Hart a. d. Alz aus dem voralpinen Raum zwischen Ostfrankreich und Böhmen (Poing/ Grab 1, Königsbronn, Milavče/Hügel C/4, Horušany, Eberfing, Korntal-Münchingen, Straubing/Grab 26, *Sémoutiers?), die der frühen bis älteren Urnenfelderzeit (BD-HA1) angehören (Pankau 2013a). In der mittleren und beginnenden jüngeren Urnenfelderzeit (HA2-HB1) nimmt die Anzahl der Gräber mit Rohmetall ab, mit den alpinen und voralpinen Gräbern von Marzoll (Grab 2), Volders (Gräber 239, 256, 305), Vomp (Grab 3), München-Unterhaching (Grab 30), Barbing (Grab 2), Regensburg-Weichs (Grab 3), Aberzhausen und Möckmühl ist eine räumliche Kontinuität gegeben. Diese signifikante Abnahme entspricht der Abnahme von Rohmetall in den zeitgleichen Hortfunden in der Zone nordwärts der Alpen. Das Fundbild der Gräber mit Rohmetall verdichtet sich im Unterinngebiet in der Nähe der Schwazer Bergbauregion, wenn wir die Barrenfunde aus dem Gräberfeld von Völs unweit von Volders (Kasseroler 1959) und Vomp (Sölder 2012) gelegen - hinzunehmen (Abb. 3). In späturnenfelderzeitlichen Gräbern (HB3) ist bisher die Beigabe von Rohmetall unbekannt, sodass das ältereisenzeitliche Grab vom Salzbergbauort Hallstatt (Grab 797) den Abschluss dieser Reihe bildet. Es ist von Bedeutung, dass innerhalb des 
Verbreitungsgebietes der bronzezeitlichen Gusskuchen in Hortfunden (Nessel 2017, 170, Abb. 1) es außer der alpinen und voralpinen Region keine weiteren Grabfunde mit Rohmetall als Beigabe bekannt sind, auch nicht in vergleichbar reich ausgestatteten Gräbern Europas, wie z. B. in der mitteldanubischen Čaka-Gruppe oder in der nordischen Bronzezeit.

\section{ZUM SOZIALEN KONTEXT}

\section{Alter und Geschlecht}

Von nur wenigen Gräbern mit Rohmetall als Beigaben wurden Alter und Geschlecht der Toten anthropologisch bestimmt. Männer sind die Toten von Ilvesheim (,älterer Mann") und Poing, Grab 1 („,frühadult", „zierlich“), angeblich eine Frau die Tote von Marzoll (ca. 18-20 Jahre alt, also frühadult). Nach archäologischen Kriterien stehen die urnenfelderzeitlichen Schwertgräber von Milavče (Hügel C/4: mit Lanzenspitze), Poing (mit Pfeilspitzen, Pferdegeschirr, Rasiermesser), Korntal-Münchingen (zwei Schwerter, Lanzenspitze), Möckmühl und München-Unterhaching (Grab 30) sowie die Lanzengräber von Königsbronn und *Kronberg (mit Pfeilspitzen) in einem betont kriegerisch-männlichen Kontext. Dies gilt auch für die mittelbronzezeitlichen Gräber mit Rohmetall, wie die waffenführenden Gräber von Kühndorf (Dolch, Beil, Pfeilspitze) und *Weischau (Beil, Dolch), während bei dem reichlich schmuckführenden Grab von Eberfing als einzige Waffe nur eine Pfeilspitze vorhanden ist. Das Messer-/Rasiermessergrab von Vomp könnte das Grab eines Schwertträgers gewesen sein, wenn wir den Doppelknopf zum Waffengurt rechnen (Jockenhövel 1971, 80; unentschieden: Clausing 2005, 43 ff.; Willms 2017, 61).

\section{Rang}

Alle verwertbaren Kriterien deuten für die Mehrzahl der Gräber mit Rohmetall darauf hin, dass die Bestatteten einer sozial herausragenden Schicht von Kriegern/Männern angehören. Dies gilt nicht nur für die Waffengräber im Allgemeinen, sondern insbesondere für die deutliche Zugehörigkeit der Rohmetallgräber zu den besonders prunkvollen früh- und älterurnenfelderzeitlichen Gräbern mit pferdegezogenen Zeremonialwagen der Art Poing/ Hart a. d. Alz (Königsbronn, *Kronberg, Milavče, Poing). Die Forschung ist sich einig, dass gerade sie die Spitze des frühurnenfelderzeitlichen Gesellschaftkegels bilden (Clausing 2005, 87 ff., 130; Pankau 2013a; 2013b; Sperber 1999; Winghart 1999; 2003). Das
Wagengrab von Poing ist derzeit das reichste frühurnenfelderzeitliche Grab Zentralmitteleuropas. Aber auch andere Kriterien, wie der Aufwand für die Grablege, wie die (über)mannslangen Steinkistengräber von Volders und das mit Steinen ausgekleidete Grab von Vomp (eventuell mit einer Steinüberhügelung; Sölder 2012), können herangezogen werden. Das waffenlose Grab mit Rohmetall und Gewichten von Horušany hebt sich durch seine Grablege im größten Hügel der Gräbergruppe von seiner Umgebung ab. Straubing (Grab 26) lieferte als exzeptionelle Beigabe den Rest eines einzinkigen Fleischhakens, der bei Homers Schilderungen ähnlichen Festen und Gelagen eine Rolle spielte (Schefzik 2009; zu Westeuropa vgl. Bowman/Needham 2007; Gerloff 2010).

\section{Zur Funktion des Rohmetalls in Gräbern}

Rohmetall steht als zum Einschmelzen vorgesehene Objektgruppe in der chaîne opératoire der Metallarbeit zwischen Gewinnung (Bergbau)/ Verhüttung, Guss und Weiterverarbeitung. $\mathrm{Ob}$ die Gräber mit Rohmetall den bronzezeitlichen Handwerker ebenso wie solche mit Gießformen oder Werkzeugen kennzeichnen, ist nach wie vor umstritten (Hansen 1991, 140 ff.; 1994, 232; Jockenhövel 2018, 220). Dass Rohmetall als Beigabenersatz, als pia fraus, für gerade nicht zur Verfügung stehende Fertigprodukte diente (Wiegel 1994, 150), ist im Einzelfall möglich, jedoch weniger wahrscheinlich, denn dann müsste Rohmetall weit häufiger als Grabbeigabe vorliegen.

Die Gräber von Volders, Vomp und *Völs liegen inmitten der Nordtiroler Bergbau- und Verhüttungsregion (Stöllner/Oeggl Hrsg. 2015). Daher sehe ich diese Gräber in einem direkten funktionalen und sozialen Kontext mit dem im Tiroler Unterinntal umgehenden Bergbau und mit der Verhüttung. Dazu gehören auch die gelegentlich vorkommenden Erzbrocken in Gräbern von Volders (Jockenhövel 1982; Sperber 2004, 305 ff.). Rohmetall in diesen Gräbern kann als Beleg für Gewinnung und Vertrieb des Rohmetalls außerhalb der Bergbauregion gelten (Sperber 2004; Winghart 1998; 1999). Die übrigen Gräber liegen abseits der klassischen ostalpinen Lagerstättenregionen. $\mathrm{Ob}$ in diesem Fall regionale oder andere Lagerstätten genutzt wurden, ist noch zu erforschen.

In den Gräbern mit Rohmetall steht die Kombination von Rohmetall und Werkzeug hinter der mit Sicheln (Barbing, Königsbronn, Marzoll, München-Unterhaching, Poing, Regensburg-Weichs, Straubing) und Gewichten zurück ( ${ }^{*}$ Kronberg mit mehreren Meißelschneiden, Lachen-Speyerdorf 
mit kleinem Blockamboss für Goldschmiedearbeit: Armbruster u. a. 2019; Hallstatt, Grab 797 mit Pickelspitze als Rest eines Bergbaugezähes).

Rohmetall kann jedoch über seine primäre Funktion hinaus weitere ökonomische und soziale Bereiche verkörpern, wie als Wertmesser, was vor allem für die plankonvexen Gusskuchen und ihre gezielten Stückelungen gelten kann. Beispielhaft ist der Hortfund mit ca. $50 \mathrm{~kg}$ Rohmetall aus der Talsiedlung (unterhalb der Höhensiedlung "Rachelburg") bei Flintsbach (Ldkr. Rosenheim, Oberbayern; Möslein/Winghart 2001) mit ganzen, dreiviertel-, halb- und viertelgroßen Exemplaren (Abb. 4; Winghart 1998, 111, Abb. 2). Mit diesen aufeinander bezogenen Maß- und Gewichtsgrößen der plankonvexen Gusskuchen bzw. „Rundkuchen“ (Terminus nach E. J. Haeberlin) erfüllt diese Serie von aes rude bzw. aes formatum fast alle Kriterien an ein umlauffähiges „Nutzgeld“ (Haeberlin 1910, 4-7).

Der augenfälligen Kombination von Rohmetall und Gewichten in einigen reichen Kriegergräbern (Horušany, Königsbronn, Milavče, Poing) kommt eine besondere Bedeutung $\mathrm{zu}$, so dass ich diese Fundgruppen nicht ausschließlich für symbolische Beigaben halte, sondern sie mit der sozialen
Position und Rolle ihrer ehemaligen Besitzer im realen Wirtschaftsleben in Beziehung setze, wie Beschaffung, Kontrolle und Verteilung der Rohstoffe. L. Rahmstorf spricht sogar von „(gewinnorientiertem) Handel durch professionelle Händler" (Rahmstorf 2016, 307), zu deren Arbeitsinstrument Waagen und ihre „Händlertaschen” gehören (zu tool kits vgl. Jockenhövel 2019a). Als Analogie können wikingerzeitliche „Krieger-Händler"-Gräber mit ihren Beigaben von Waffen und Waagen im Baltikum dienen (Apals/Apala 1994).

Prüfen, Messen und Wägen sind Grundelemente jedes Wirtschaftslebens. Wenn mittlerweile auch aus der ausgehenden Mittelbronzezeit und zu Beginn der jüngeren Bronzezeit des ,,barbarischen“ Europas entsprechende Fundgruppen vorliegen, wird ihre überregionale Einbindung in einen altweltlichen Kultur- und Wirtschaftsraum, der die bronzezeitlichen Hochkulturen einbezieht, besonders deutlich (Rahmstorf 2019). Wir können voraussetzen, dass zumindest die bronzezeitlichen „Kaufleute“ (und „Reisenden": Kristiansen/Larsson 2005) bei kompatiblen Maßsystemen sich gegenseitig kontrollierten, vertrauten und, wenn notwendig über Dolmetscher, verständigten (Jockenhövel 2019b, 30, Abb. 5).
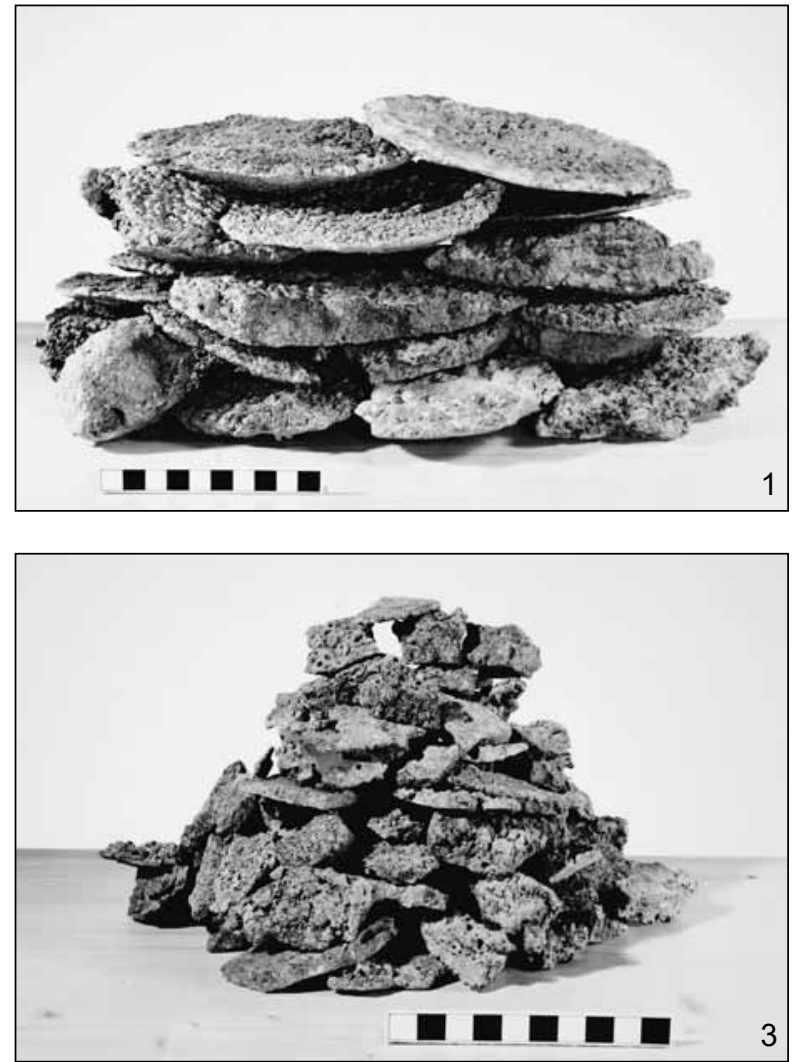
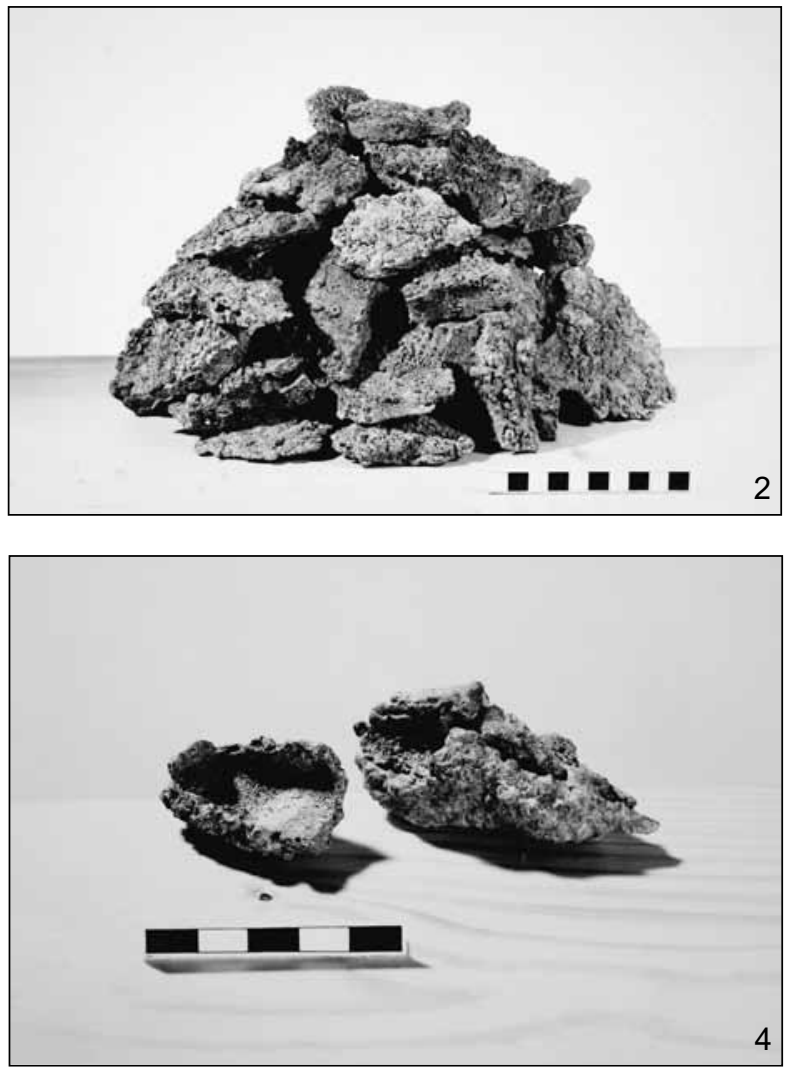

Abb. 4. Flintsbach (Ldkr. Rosenheim). Urnenfelderzeitliche Siedlung im Tal unterhalb der Höhensiedlung „Rachelburg“. Vollständige und portionierte Gusskuchen aus Kupfer (nach Winghart 1998, Abb. 2). 
Die bronzezeitlichen Metallhandwerker hatten bereits enormes physikalisch-chemisches Wissen in der Metallurgie empirisch angehäuft, das sie auf Grund von bestimmten Kriterien (wie Härte, Duktilität, Gewicht, Farbe, Klang, Geruch, Toxizität der Metalle) befähigte, spezifische Eigenschaften und den Wert von Rohmetall einzuschätzen. Gerade die Bruchstücke von Rohmetall ermöglichten dem Metallhandwerker eine Inaugenscheinnahme ihres „Inneren“ und damit eine erste Qualitätsbeurteilung des Rohmetalls vorzunehmen. Die besonderen Legierungen der Barren waren an ihrer Farbe schon äußerlich erkennbar. Unterschiedliche Kupfersorten wurden bereits in den bronzezeitlichen Hochkulturen namentlich benannt: So unterschieden z. B. die Hethiter "gewöhnliches" vom "guten" Kupfer mit entsprechender fester Wertrelation zu Gold und Silber (Alparslan/Doğan-Alparslan 2011). Diese Sorten können in schriftlosen Bronzezeit-Kulturen durchaus mit modernen Metall-Analysengruppen vergleichen werden (z. B. Arsen-, Kupferkies- oder Fahlerzbronzen).

Bei aller Kontrolle war man jedoch vor Betrug nicht geschützt. Um 2000 v. Chr. wurden im Oman (Teil des alten Metalllandes Magan/Makkan) plankonvexe Gusskuchen mit Schlacke gefüttert (Weisgerber/Yule 2003). Im kroatischen jungbronzezeitlichen Hort von Miljana enthielt ein Gusskuchen im Innern einen Kern aus Blei und aus ungarischen Horten liegen hohle Gusskuchen vor (Hansen 1994, 232). Im 8. Jh. v. Chr. klagte der alttestamentarische Prophet Micha (Micha 6, 11): „Soll ich die gefälschte Waage ungestraft lassen und den Beutel mit den falschen Gewichten?".

Rohmetall diente bei den Leichenspielen für Patroklos als Preisgeld. Der als Preis ausgesetzte Diskos aus Eisen (Homer, Ilias 23, 826 ff.) war so schwer, dass er für fünf (!) Jahre den Eisenbedarf eines frühgriechischen oíkos decken konnte. Archäologisch fassbar ist eine solche zeitgenössische Scheibe aus Schmiedeeisen (ca. $5 \mathrm{~kg}$ schwer) im Riesentumulus III der phrygischen Königsstadt Gordion (Yassı Hüyük; Abb. 1: 25), wo sie mit einem weiteren Eisenbarren-Fragment (ca. 2,7 kg schwer) zu den Mitgaben einer königlichen Bestattung gehört (Körte/Körte 1904, 79, Abb. 69: a).

Wir können zusammenfassen: Rohmetall in unterschiedlicher Form ist in bronzezeitlichen Gräbern eine sehr seltene Beigabe, wenn man bedenkt, dass unter zehntausenden Gräbern nur ca. 35/36 Gräbern Rohmetall überliefert ist. Dies gilt auch für andere Fundgruppen aus dem Bereich der Metallarbeit. Die ältesten Gräber datieren in die Mittelbronzezeit, ein deutlicher Schwerpunk liegt in der frühen und älteren Urnenfelderzeit Zentraleuropas. Dies korrelliert mit der Häufigkeit der "Brucherz"-Hortfunde mit Rohmetall. In dieser Zeit konzentriert sich das Vorkommen dieser Gräber auf die Lagerstätten von Kupfererzen im Inntal und auf die voralpine Region zwischen Alpen und Donau als Distributionsraum von Rohmetall. In vielen Gräbern spiegelt sich - bis auf eine Ausnahme (Marzoll, Grab 2 - Nr. 5) - eine männlich-kriegerische Sphäre. Möglicherweise zeichnet sich in der Kombination von Rohmetall mit Gewichten in einigen reich ausgestatteten Gräbern eine Personalunion von Kriegern und "Händlern“ ab. Da Rohmetall (mit Ausnahme der Barren) in den Gräbern fast ausschließlich als Bruch, jedoch nie vollständig überliefert ist, liegt auch eine symbolische Bedeutung nahe. Rohmetall hatte einen besonderen Wert. So war Rohmetall als Preis bei den Leichenspielen homerischer Heroen ausgesetzt und vor Betrugsabsichten nicht sicher. 


\section{LITERATUR}

Adler 1990 - H. Adler: Fundberichte 1990: KG Kronberg. Fundberichte aus Österreich 29, 1990, 201-202.

Alparslan/Doğan-Alparslan 2011 - M. Alparslan/M. Doğan-Alparslan: Symbol der ewigen Herrschaft: Metall als Grundlage des hethitischen Reiches. In: Ü. Yalçın (Hrsg.): Anatolian Metal V. Der Anschnitt 24. Bochum 2011, 79-84.

Apals/Apala 1994 - J. Apals/Z. Apala: Der lettgallische Krieger und Kaufmann in der Wikingerzeit (nach den Materialien des Gräberfeldes Ģūgeri). In: B. Stjernquist (ed.): Prehistoric graves as a source of information. Symposium at Kastlösa, Öland, 1992. Konferenser 29. Stockholm 1994, 93-110.

Armbruster u. a. 2019 - B. Armbruster/A. Jockenhövel/ A. Kapuran/R. Ramadanski: The moulds from Velebit and European Bronze Age metal anvils. Starinar 69, 2019, 139-182.

Bachmann/Jockenhövel 1974 - H.-G. Bachmann/A. Jockenhövel: $\mathrm{Zu}$ den Stabbarren aus dem Rhein bei Mainz. Archäologisches Korrespondenzblatt 4, 1974, 139-144.

Bachmann u. a. 2003 - H.-G. Bachmann/A. Jockenhövel/ U. Spichal/G. Wolf: Zur bronzezeitlichen Metallversorgung im mittleren Westdeutschland: Von der Lagerstätte zum Endprodukt. Berichte der Kommission für archäologische Landesforschung in Hessen 7, 2003, 67-120.

Berger 1984 - A. Berger: Die Bronzezeit in Ober- und Mittelfranken. Materialhefte zur bayerischen Vorgeschichte 52. Kallmünz/Opf. 1984.

Bowman/Needham 2007 - Sh. Bowman/S. Needham: The Dunaverney and Little Thetford flesh-hooks: history, technology and their position within the later Bronze Age Atlantic Zone feasting complex. The Antiquaries Journal 87, 2007, 53-108.

Clausing 2005-C. Clausing: Untersuchungen zu den urnenfelderzeitlichen Gräbern mit Waffenbeigaben vom Alpenkamm bis zur Südzone des Nordischen Kreises. Eine Analyse ihrer Grabinventare und Grabformen. BAR 1375. Oxford 2005.

Drescher 1976 - H. Drescher: Barren. In: Reallexikon zur Germanischen Altertumskunde 2. Berlin - New York 1976, 60-70.

Ebner 2001 - K. Ebner: Die mittlere Bronzezeit in Südthüringen. Unpublizierte Dissertationsarbeit. Philipps-Universität Marburg. Marburg 2001.

Feustel 1993 - R. Feustel: Zur bronzezeitlichen Hügelgräberkultur in Südthüringen. Alt-Thüringen 27, 1993, 53-123.

Gerloff 2010 - S. Gerloff: Atlantic cauldrons and buckets of the Late Bronze and Early Iron Ages in Western Europe. PBF II/18. Stuttgart 2010.

Görner 2003 - I. Görner: Die Mittel- und Spätbronzezeit zwischen Mannheim und Karlsruhe. Fundberichte aus Baden-Württemberg 27, 2003, 79-279.

Haeberlin 1910 - E. J. Haeberlin: Aes grave. Das Schwergeld Roms und Mittelitaliens einschließlich der ihm vorausgehenden Rohbronzewährung 1. Frankfurt am Main 1910.

Hansen 1991 -S. Hansen: Studien zu den Metalldeponierungen während der Urnenfelderzeit im Rhein-Main-Gebiet. UPA 5. Bonn 1991.

Hansen 1994 -S. Hansen: Studien zu den Metalldeponierungen während der älteren Urnenfelderzeit zwischen Rhônetal und Karpatenbecken. UPA 21. Bonn 1994.

Hell 1948 - M. Hell: Funde der Bronzezeit und Urnenfelderkultur aus Marzoll, Ldkr. Berchtesgaden. Bayerische Vorgeschichtsblätter 17, 1948, 23-36.
Hennig 1993 - H. Hennig: Urnenfelder aus dem Regensburger Raum. Materialhefte zur bayerischen Vorgeschichte 65. Kallmünz/Opf. 1993.

Hundt 1964 - H.-J. Hundt: Katalog Straubing II. Die Funde der Hügelgräberbronzezeit und der Urnenfelderzeit. Materialhefte zur bayerischen Vorgeschichte 19. Kallmünz/ Opf. 1964.

Jockenhövel 1971 - A. Jockenhövel: Die Rasiermesser in Mitteleuropa (Süddeutschland, Tschechoslowakei, Österreich, Schweiz). PBF VIII/1. München 1971.

Jockenhövel 1973 - A. Jockenhövel: Urnenfelderzeitliche Barren als Grabbeigaben. Archäologisches Korrespondenzblatt 3, 1973, 23-28.

Jockenhövel 1982 - A. Jockenhövel: Zeugnisse der primären Metallurgie in Gräbern der Bronze- und Alteisenzeit Mitteleuropas. Archeologia Polski 27, 1982, 293-301.

Jockenhövel 2018 - A. Jockenhövel: Alteuropäische Gräber der Kupferzeit, Bronzezeit und Älteren Eisenzeit mit Beigaben aus dem Gießereiwesen (Gießformen, Düsen, Tiegel). In: M. Overbeck (Hrsg.): Die Gießformen in West- und Süddeutschland (Saarland, Rheinland-Pfalz, Hessen, Baden-Württemberg, Bayern). PBF XIX/3. Stuttgart 2018, 213-317.

Jockenhövel 2019a - A. Jockenhövel: Bronzezeitliche Werkzeugsätze als Anzeichen spezialisierter Handwerker. Plattform. Zeitschrift des Vereins für Pfahlbau- und Heimatkunde e. V. 25-27, 2019, 89-110.

Jockenhövel $2019 b$ - A. Jockenhövel: Nemo nascitur artifex. Zum Kontext bronzezeitlichen Wissens. In: D. Neumann/G. Woltermann/R. Gleser (Hrsg.): Spezialisierungen in der Bronzezeit. Archäologische Quellen und Modelle. Beiträge zur Sitzung der AG Bronzezeit auf der 83. Jahrestagung des Nordwestdeutschen Verbandes für Altertumsforschung 18.-21. September 2016 in Münster. Neolithikum und ältere Metallzeiten. Studien und Materialien 4. Münster 2019, 9-42.

Kasseroler 1959 - A. Kasseroler: Das Urnenfeld von Volders. Schlern-Schriften 204. Innsbruck 1959.

Körte/Körte 1904 - G. Körte/A. Körte: Gordion. Ergebnisse der Ausgrabung im Jahre 1904 (mit einem Anhang von R. Kobert). Jahrbuch des Kaiserlichen Archäologischen Instituts 5. Ergänzungsheft. Berlin 1904.

Koschik 1981 - H. Koschik: Die Bronzezeit im südwestlichen Oberbayern. Materialhefte zur bayerischen Vorgeschichte 50. Kallmünz/Opf. 1981.

Kristiansen/Larsson 2005 - L. Kristiansen/T. B. Larsson: The rise of Bronze Age society. Travels, transmissions and transformations. Cambridge 2005.

Kromer 1959 - K. Kromer: Das Gräberfeld von Hallstatt. Firenze 1959.

Ludwig-Lukanow 1983 - S. Ludwig-Lukanow: Hügelgräberbronzezeit und Urnenfelderkultur im Nördlinger Ries. Materialhefte zur bayerischen Vorgeschichte 48. Kallmünz/Opf. 1983.

Modl 2010 - D. Modl: Zur Herstellung und Zerkleinerung von plankonvexen Gusskuchen in der spätbronzezeitlichen Steiermark, Osterreich. In: F. Both (Hrsg.): Experimentelle Archäologie in Europa. Bilanz 2010. Heft 9. Oldenburg 2010, 127-151.

Modl 2019 - D. Modl: Recording plano-convex ingots (Gusskuchen) from Late Bronze Age Styria and Upper Austria - A short manual for the documentation of morphological and technological features from production 
and partition. In: R. Tourk/Th. Stöllner/G. Goldenberg (eds.): Alpine Copper II - Alpenkupfer II - Rame delle Alpi II - Cuivre des Alpes II. New results and perspectives on prehistoric copper production. Der Anschnitt 42. Rahden/ Westf. 2019, 373-398.

Möslein/Winghart 2001 - St. Möslein/St. Winghart: Die Rachelburg bei Flintsbach a. Inn, Lkr. Rosenheim, Oberbayern. Berichte Bayerischen Bodendenkmalpflege 39-40, 1998-1999 (2001), 145-348.

Mozsolics 1981 - A. Mozsolics: Gußkuchen aus wieder eingeschmolzenem Altmetall. In: H. Kaufmann (Hrsg.): Beiträge zur Ur- und Frühgeschichte I. Festschrift Werner Coblenz. Arbeits- und Forschungsberichte zur Sächsischen Bodendenkmalpflege 16. Berlin 1981, 403-417.

Mozsolics 1984 - A. Mozsolics: Ein Beitrag zum Metallhandwerk der ungarischen Bronzezeit. Bericht der Römisch-Germanischen Kommission 65, 1984, 19-72.

Nessel 2017 - B. Nessel: Von warmen und kalten Brüchen. Bruchmuster und Konzepte der Portionierung bronzezeitlichen Rohmaterials am Beispiel plankonvexer Gusskuchen. In: D. Brandherm/B. Nessel (Hrsg.): Phasenübergänge und Umbrüche im bronzezeitlichen Europa. Beiträge zur Sitzung der Arbeitsgemeinschaft Bronzezeit auf der 80. Jahrestagung des Nordwestdeutschen Verbandes für Altertumsforschung. UPA 297. Bonn 2017, 169-198.

Pankau 2013a - C. Pankau: Neue Forschungen zu den Wagengräbern der Hart an der Alz-Gruppe. In: L. Husty/ K. Schmotz (Hrsg.): Vorträge des 31. Niederbayerischen Archäologentages. Rahden/Westf. 2013, 113-147.

Pankau 2013b - C. Pankau: Das spätbronzezeitliche Wagengrab von Königsbronn (Lkr. Heidenheim). Mit Beiträgen von Josef Riederer und Johannes Behringer. Jahrbuch des Römisch-Germanischen Zentralmuseums Mainz 60, 2013, 1-103.

Pare 1999 - C. F. E. Pare: Weights and weighing in Bronze Age Central Europe. In: V. L. Aravantinos/J. A Barceló/ Chr. Bockisch-Bräuer (Hrsg.): Eliten in der Bronzezeit. Teil 2. Ergebnisse zweier Kolloquien in Mainz und Athen. Monographien des Römisch-Germanischen Zentralmuseums Mainz 43. Mainz 1999, 421-514.

Primas/Pernicka 1998 - M. Primas/E. Pernicka: Der Depotfund von Oberwilflingen: neue Ergebnisse zur Zirkulation von Metallbarren. Germania 76, 1998, 25-62.

Rahmstorf2016 - L. Rahmstorf: Die Rahmenbedingungen des bronzezeitlichen Handels in Europa und im Alten Orient einschließlich Ägyptens. In: U. L. Dietz/A. Jockenhövel (Hrsg.): 50 Jahre "Prähistorische Bronzefunde". Bilanz und Perspektiven. PBF XX/14. Stuttgart 2016, 291-310.

Rahmstorf 2019 - L. Rahmstorf: Scales, weights and weight-regulated artefacts in Middle and Late Bronze Age. Antiquity 93, 2019, 1-14.

Reiter/Linke 2016 - V. Reiter/R. Linke: Ein Werkplatz mit Brucherzdepot der ausgehenden Bronzezeit aus Rannersdorf, Niederösterreich. Fundberichte aus Österreich 55, 2016, 144-182.

Salaš 2018 - M. Salaš: Kovová depozita mladší doby bronzové $z$ hradiska Réna u Ivančic $=$ Metalldeponierungen der älteren Urnenfelderzeit von der Wallanlage Réna bei Ivančice. Brno 2018.

Schefzig 2009 - M. Schefzig: Ein urnenfelderzeitlicher Fleischhaken von Heimstetten bei München - Verlust oder Opfer? In: J. M. Bagley/Ch. Eggl/D. Neumann/ M. Schefzik (Hrsg.): Alpen, Kult und Eisenzeit. Festschrift für Amei Lang zum 65. Geburtstag. Internationale Archäologie. Studia Honoraria 30. Rahden/Westf. 2009, 157-165.

Schwerin von Krosigk 1976 - H. Schwerin von Krosigk: Untersuchungen zum vor- und frühgeschichtlichen Siedlungsablauf am Fundbild von Bornhöved, Gönnebek, Groß Kummerfeld und Schmalensee, Kr. Segeberg/Holstein. Offa-Ergänzungs-Reihe 1. Schleswig 1976.

Sölder 2012 - W. Sölder: Spätbronzezeitliches Brandgräberfeld Vomp/Fiechter Au. In: Amt der Tiroler Landesregierung, Kulturabteilung (Hrsg.): Kulturberichte aus Tirol 2012. 63. Denkmalbericht. Innsbruck 2012, 231-236.

Sperber 1999 - L. Sperber: Zu den Schwertträgern im westlichen Kreis der Urnenfelderkultur. Profane und religiöse Aspekte. In: V. L. Aravantinos/J. A Barceló/ Chr. Bockisch-Bräuer (Hrsg.): Eliten in der Bronzezeit. Teil 2. Ergebnisse zweier Kolloquien in Mainz und Athen. Monographien des Römisch-Germanischen Zentralmuseums Mainz 43. Mainz 1999, 605-659.

Sperber 2000 - L. Sperber: Zum Grab eines spätbronzezeitlichen Metallhandwerkers von Lachen-Speyerdorf, Stadt Neustadt a. d. Weinstrasse. Archäologisches Korrespondenzblatt 30, 2000, 383-402.

Sperber 2004 - L. Sperber: Zur Bedeutung des nördlichen Alpenraumes für die spätbronzezeitliche Kupferversorgung in Mitteleuropa mit besonderer Berücksichtigung Nordtirols. In: G. Weisgerber/G. Goldenberg (Hrsg.): Alpenkupfer - Rame delle Alpi. Der Anschnitt. Beiheft 17. Bochum 2004, 303-345.

Stöllner/Oeggl Hrsg. 2015 - Th. Stöllner/K. Oeggl Hrsg.: Bergauf Bergab. 10.000 Jahre Bergbau in den Ostalpen. Wissenschaftlicher Beiband zur Ausstellung im Deutschen Bergbau-Museum Bochum vom 31.10.2015-24.04.2016, im Vorarlberg Museum Bregenz vom 11.06.2016-26.10.2016. Veröffentlichung aus dem Deutschen Bergbau-Museum Bochum 207. Bochum 2015.

Weisgerber/Yule 2003 - G. Weisgerber/P. Yule: Al-Aqir near Bahlā - an Early Bronze dam site with planoconvex 'copper' ingots. Arabian Archaeology and Epigraphy 14, 2003, 24-53.

Wiegel 1992 - B. Wiegel: Trachtkreise im südlichen Hügelgräberbereich. Studien zur Beigabensitte der Mittelbronzezeit unter besonderer Berücksichtigung forschungsgeschichtlicher Aspekte. Band 2/1: Kommentiertes Fundortverzeichnis der Flachgräber und Grabhügel. Internationale Archäologie 5/2. Espelkamp 1992.

Wiegel 1994 - B. Wiegel: Trachtkreise im südlichen Hügelgräberbereich. Studien zur Beigabensitte der Mittelbronzezeit unter besonderer Berücksichtigung forschungsgeschichtlicher Aspekte. Band 1: Auswertung. Beilagen. Internationale Archäologie 5/1. Espelkamp 1994.

Willms 2017 - Ch. Willms (aus dem Nachlass bearbeitet von Albrecht Jockenhövel): Das Steinkistengrab von Bad Nauheim. Stand der Auswertung im Jahre 2015. In: P. Fasold/L. Giemsch/K. Ottendorf/D. Winger (Hrsg.): Forschungen in Franconofurd. Festschrift für Egon Wamers zum 65. Geburtstag. Regensburg 2017, 55-76.

Winghart 1998 -St. Winghart: Produktion, Verarbeitung und Verteilung. Überlegungen zur Bedeutung metallischer Rohstoffe bei der Ausbildung politischer Systeme im südbayerischen Alpenvorland während der Bronzezeit. In: Th. Busset/J. Mathieu (éds.): Mobilité spatiale et frontières $=$ Räumliche Mobilität und Grenzen . Histoire 
des Alpes - Storia delle Alpi - Geschichte der Alpen 3. Zürich 1998, 107-122.

Winghart 1999 - St. Winghart: Die Wagengräber von Poing und Hart a. d. Alz. Evidenz und Ursachen spätbronzezeitlicher Elitenbildung in der Zone nordwärts der Alpen. In: V. L. Aravantinos/J. A Barceló/Chr. Bockisch-
Bräuer (Hrsg.): Eliten in der Bronzezeit. Teil 2. Ergebnisse zweier Kolloquien in Mainz und Athen. Monographien des Römisch-Germanischen Zentralmuseums Mainz 43. Mainz 1999, 515-532.

Winghart 2003 -St. Winghart: Poing. In: Reallexikon zur Germanischen Altertumskunde 23. Berlin-New York 2003, 239-243.

Manuskript angenommen am 1.9. 2020

Prof. Dr. Albrecht Jockenhövel

Am Biederlackturm 7

Abstract translated by Albrecht Jockenhövel

D - 48282 Emsdetten

Súhrn preložila Anita Kozubová

jockenh@uni-muenster.de

\title{
Hroby z doby bronzovej so surovým kovom: skupina nálezov medzi praktickou funkciou a symbolickým významom
}

\author{
Albrecht Jockenhövel
}

\section{SÚHRN}

Surový kov v podobe rôznych foriem ingotov je dôležitým článkom $v$ chaîne opératoire spracovania kovov $v$ dobe bronzovej (tažba medenej rudy, tavenie, hotové výrobky). Počiatky tejto tradície pozorujeme na základe nálezov v strednej dobe bronzovej (BB-BC) a svoj vrchol dosahuje v staršej fáze kultúr popolnicových polí (BD-HA) výskytom tzv. surovinových depotov. Je zarážajúce, že surový kov nehral takmer žiadnu úlohu $\mathrm{v}$ tisícoch hrobov $\mathrm{z}$ doby bronzovej v Európe, kde bol doteraz nájdený iba v 35 hroboch, a to výlučne v strednej Európe (najmä v Tirolsku, Bavorsku, Čechách) v alpskej oblasti a na severnom úpätí Ápl. Mimo uvedených regiónov porovnatelné hrobové celky so surovým kovov nenachádzame ani v severskej dobe bronzovej, ani napríklad v stredodunajskej čakanskej kultúre.
Kombinácia surového kovu a d’alších nálezov spája predmetné hroby takmer výlučne s mužskou sférou. Jedinou výnimkou je ženský hrob 2 z Marzollu. Väčšina hrobov je bohato vybavená zbraňami (meče, hroty oštepov, dýky atd.) a vozmi (Königsbronn, Milavče, Poing).

Pomerne častá kombinácia s kosákmi a váhami ukazuje na pol'nohospodárske aktivity a štandardizovaný hodnotový systém. Koncentrácia hrobov obsahujúcich surový kov v blízkosti východoalpských ložísk medenej rudy (Tirolsko, Salzburgsko) môže naznačovał tažbu, kontrolu a distribúciu surového kovu bojovníkmi a „obchodníkmi“. Surový kov mal pravdepodobne okrem primárnej funkcie aj d’alšie využitie, napr. ako hodnotová miera, čo je zrejmé v prípade plankonvexných ingotov a ich cieleného rozdelenia. 
